\title{
A Novel Algorithm to Select Cluster Heads with Highes and Balanced Energy in Wireless Sensor Networks
}

\author{
Abdo Saif Mohammed, \\ Department of Electronics and Communication \\ Sri Jayachamarajendra Colleges of Engineering \\ Mysore, Karnataka, India.
}

\begin{abstract}
A wireless sensor network consists of large number of sensor nodes limited by a small energy spread in a large geographical area. Many algorithm collect information from the network by using clustering. LEACH is the most famous and popular one from this algorithm to maintain the energy efficiency of sensor nodes. In this paper, we propose a new algorithm to choose the cluster head with the highest energy. In our proposed work, we have collected all the nodes in the array and arranged them in descending order and then we select the best three nodes with the highest energy as a main cluster heads.
\end{abstract}

\section{Keywords}

Wireless sensor network, clustering, lifetime.

\section{INTRODUCTION}

The sensor nodes in the wireless sensor network can send information and cooperate with each other to accomplish some specific tasks through the application of communication for wireless self-organization [1]. Moreover wireless sensor network can be used in many areas, such as the environmental ,medical , agriculture, industry and military monitoring [2]. Because of size, cost and other factors of sensors, each sensor node has an extreme limitation on the bandwidth and the capacity of the battery. And usually the sensor nodes are arranged in very bad condition. In this case, to design any protocol must take into account the limited energy of nodes. A large number of experiments Showed that the strategy of cluster is the main solution to this issue. If we looked in the numerous clustering algorithms, Low-Energy Adaptive Clustering (LEACH) [3] is one of the popular and important algorithm to improve Clustering in WSN.. Many of clustering algorithms [3], [4], [5] rely on Leach algorithm is largely dependent. However, the LEACH algorithm mainly depends on the randomly selected cluster-heads $(\mathrm{CH})$, without network energy consumption and to enhance the life time.

\section{RELATED WORK}

In 2000 Heinzelman [6] designed and implemented Low-Energy Adaptive Clustering Hierarchy (LEACH) protocol for WSN and in 2004 [7], he assumed the BS is located at thecenter of the sensor fields. Lai [8] in 2007 proposed a genetic algorithm to find an approximate solution for the NP-complete Disjoint Set Covers (DSC) problem and in the same year Chamam [9] addressed the problem of maximizing the WSN lifetime under the area coverage constraint. Later in 2007 [10],[11] strategy of important initial energy (IEA), which increases WSN lifetime that provide different levels of initial energy to a different sensor nodes and a new clustering algorithm ESCAL, which is appropriate for the WSN, which decrease the distance of transmitting between cluster-heads and base station was proposed. In 2008, routing method based on the reconfiguration of an autonomous optimization of neural network dynamics, which reduces energy that used for computing an autonomous

\author{
M.N.Shanmukhaswamy \\ Department of Electronics and Communication \\ Sri Jayachamarajendra Colleges of Engineering: \\ Mysore, Karnataka, India.
}

and distributor of about $6.7 \%$ [12]. TB-LEACH was introduced [13] to improve LEACH protocol, to form constant number of clusters, by using random-timer, without any global information. A new Protocol for the filtering of the contract and avoid obstacles of LEACH protocol was proposed and different WSN protocol was studied [15] [16]. MS-Leach was combined with LEACH to form a single-hop Transmissions and multi-hop transmissions in the clusters [17].In 2009, a maximum lifetime of a data-gathering sensor network, which is defined as the number of rounds until the first node exhausted its energy, is NP-complete was achieved [18]. To overcome the weakness of the asymmetrical distribution of the cluster heads in LEACH and in selecting the head of the cluster algorithm and the onehop Routing algorithm, to extend the life of the network was suggested [19][20]. A new plan on the relationship between energy and cluster-head and coverage in sensor networks was discussed[21] and a new teaching to improve LEACH includes three stages: non-balanced clusters, uneven energy distributions, and unnecessary energy has been suggested in [22].In 2010, a program was explored [23] to find optimal clustering program in order to reduce energy consumption for the entire network and using the mathematical and statistics to prove it and a novel protocol called LEACH-B to overcome the shortcoming of the original LEACH by taking optimal cluster heads at each round and consider about residual energy of the nodes was proposed [24] and also peach algorithm that can ensure the organizing of cluster heads, modified LEACH without using the location information of nodes, LEACH-HPR to election cluster head, WLEACH without considering measuring Accuracy of data aggregation and change in LEACH to a multi-hop to reduce the energy consumption of sensor nodes was proposed [25-28].A heuristic approach to solve the problem of the accumulated data gathering in sensor networks, an improvement in LEACH protocol to overcome the shortcoming of LEACH (FZ-LEACH algorithm), -LEACH to solves the issue of node heterogeneity, Leach-SM protocol, to improve the leach using a protocol selected spare nodes as well as cluster heads and evolutionary algorithm to find an optimal cluster heads in wireless sensor network was proposed and suggested [29-33] in 2011. LEACH [3] [6] [34] is one of the most popular hierarchical routing algorithms for clustering of WSNs. Some clusters heads of leach are formed in a self-organized manner. So it is a suitable solution for the efficient use of energy in the sensor network. Although, Leach is the right solution in the field of data collection, but had some issues and several limitations. LEACH does not address the problem of closer nodes and thus redundant data may be transferred to the base station. Many $\mathrm{CHs}$ are located in a specific area that may produce clusters with low energy, because Cluster heads are not selected in a distributed manner. LEACH is a kind of adaptive algorithm to organize the nodes into cluster, every cluster have one node as cluster head. [35][36][37]. The process is executed in periodical manner; every round consists of two phases: first phase building a cluster 
head and second phase data communication. In the first phase, close nodes make a cluster dynamically, and one node will be selected as cluster head randomly; in the second phase, every node send their data to cluster head, then cluster head collect the data and sends it to the sink node. The cluster heads consume more energy than ordinary nodes, because heads need to fuse the data and communicate with sink node. LEACH algorithm can ensure that each node in one cluster would be selected as cluster head in equal capability, which makes each node consume energy relatively equally. The requirement of selecting cluster head in LEACH is follows:

Each node produces a random number between 0 and 1 , and check it with the threshold value $\mathrm{T}$ (n)if this number is less than $\mathrm{T}(\mathrm{n})$, and then this node represents the number chooses as cluster head. When node has been cluster head, then $T(n)$ is set to 0 , to let the possibility of other nodes to select is $T(n)$. As the number of nodes which have been cluster head increases, $T(n)$ will increase, so the possibility for the rest of nodes to be selected will increase. When there is only one node left, T (n) $=1$, which means this node will be selected for sure. $\mathrm{T}(\mathrm{n})$ could be defined as follows [38]:

$$
\mathrm{T}(\mathrm{n})= \begin{cases}\frac{p}{1-p *\left(r \bmod \left(\frac{1}{p}\right)\right)} & , n \in G \\ 0 & \text { otherwise }\end{cases}
$$

Where $\mathrm{p}$ represent the desired percentage of cluster heads, $r$ represent the current round, and $\mathrm{G}$ represent the set of nodes that have not been cluster-head in the last $1 / p$ rounds. When the cluster head is selected, it will announce other nodes. Non cluster-head nodes will select a cluster to join with it, according to the distance between them and the cluster heads. When the cluster heads received all messages to share with it, they will produce a message at the time to inform all the nodes in their clusters.

\section{PROPOSED WORK}

We proposed a new algorithm to extend the lifetime of wireless sensor networks. This proposed build as follows:

\subsection{Cluster Head Selection}

We have proposed a new algorithm to choose the cluster head with the largest energy. After identifying the three main cluster heads we have build a binary tree around these three cluster heads as shown in Figure 1.

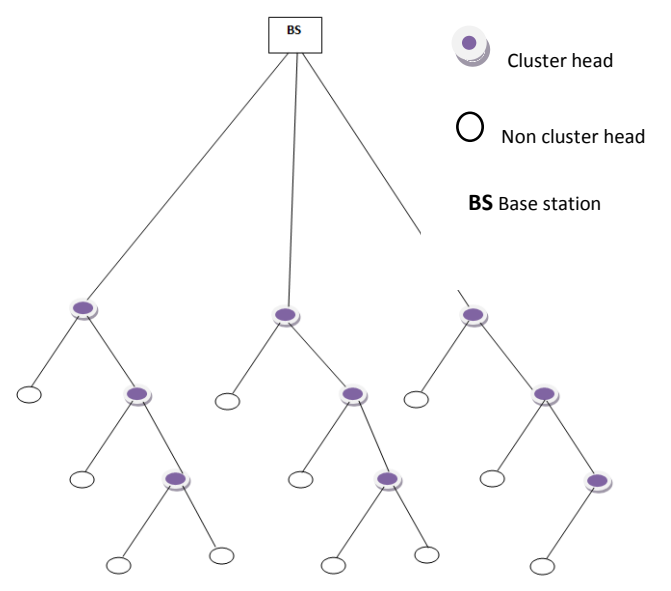

Figure 1. The Clustered Architecture with 20 nodes

Where $s 1=0$ and $s 2=1$, according to the following:

Branches of the first point were building using the $n-s 1$ (where $\mathrm{n}$-s1 represents the left side for the first point) and $\mathrm{s} 2+3$ (where s $2+3$ represent the Right side for the first point). Increase s1and s2 by one after the second step, the branches of the second Point was build using the n-s1 (where n-S1 represents the left side for the second point after increasing)and $s 2+3$ (where $s 2+3$ represents a part of right side for the second point after increasing).Increase $\mathrm{s} 1$ and $\mathrm{s} 2$ by one. At the last step, branches of the third point has been billed by using the n-s1(where n-s1 represents the left side for the third point) and $\mathrm{s} 2+3$ (represents a part of right side for the third point).If the number of nodes is more than ten, increase $\mathrm{s} 1$ and s2 by one and repeat all the five steps. From the proposed algorithm, we can build many levels with binary tree. Always right sides of tree have highest energy than left side. Because the nodes of right side will become the cluster to other nodes, in the other levels. Rebuilding the tree from the beginning of the rounds by setting $\mathrm{s} 1$ and $\mathrm{s} 2$ to 0 and 1.

\subsection{Cluster Set-up}

The proposed algorithm selects, three nodes with highest energy as cluster heads, then these three clusters heads broadcasts an advertisement messages containing information about cluster heads and it's "id". The highest and lowest nodes decides which cluster to join according to the nearest of the advertisement signal. Then the "none" cluster head sends response message, "Joint-response" to the nearest cluster head, the joint-response message contains its own id and id of the nearest cluster head it joins with it.

\subsection{Data Transmission}

Three cluster heads use TDMA schedule to collect information from all nodes and transmit it to the base station, as in LEACH.

\section{Algorithm:}

Pseudo Code of our Algorithm:

1: $\quad$ S1 10 and $s 2 \leftarrow 1$

2: Build $s(n)$ array include $n$ nodes and

Sort it as descending according to

Residual energy

3: Build $w(n)$ array include position of

$S(n)$ and sort it as descending

according to $s(n)$

4: For every $j: 3$

5: $\quad$ Select the three nodes with

High energy as clusters heads from

$S(n)$

6: For every I: $n$

7: $\quad$ For every j: 3

8: $\quad$ If $w(n-s 1) !=w(s 2+3)$

9: $\quad$ Begin

10: $\quad$ Select $s(n-s 1)$ to build

Left branch of the tree

and select $s(s 2+3)$ to

build right branch of tree

11: $\quad s 1 \leftarrow s 1+1$ and $s 2 \leftarrow s 2+1$

12: $\quad$ End

13: $\quad$ Else select $s(n-s 1)$

14: End for

15: End for

In the above algorithm the number of heads will be specified in each round, where the number of cluster heads is equal to $[(\mathrm{n} / 2)-1]$ and number of non cluster heads is equal to $[(\mathrm{n} / 2)+1]$, Where $\mathrm{n}$ is the number of nodes.

\section{SYSTEM MODEL}

We have considered the identical radio model as proposed by W.R.Heinzelman [6], the equations used to calculate 
transmission costs and receiving costs for a k-bit message for a distance $\mathrm{d}$, the energy consumed for the sender is:

$\operatorname{ETx}(L, d)=$ ETx-elec $(L)+$ ETx-amp $(L, d)$

$$
= \begin{cases}\text { LEelec }+ \text { LEfsd } d^{2} & \text { if } d<d 0 \\ \text { LEelec }+ \text { LEmpd } & \text { if } d \geq d 0\end{cases}
$$

Energy consumed for the receiver is:

ERx $(\mathrm{L})=$ ERx-elec $(\mathrm{L})=$ L.Eelec

$d=d 0=\sqrt{E f s / E m p}$

In Equation 1 and 2, parameter ETx-elec and ERx-elec means the power dissipated to run the transmitter or receiver.

\section{SEMULATION RESULTS}

Table 1. Simulation parameters

\begin{tabular}{|l|l|}
\hline Parameter & Value \\
\hline Eelect & $50 \mathrm{~nJ} / \mathrm{bit}$ \\
\hline EDA & $5 \mathrm{~nJ} / \mathrm{bit} / \mathrm{message}$ \\
\hline Efs & $10 \mathrm{pJ} / \mathrm{bit} / \mathrm{m} 2$ \\
\hline Emp & $0.0013 \mathrm{pJ} / \mathrm{bit} / \mathrm{m} 4$ \\
\hline Eo & $0.5 \mathrm{~J}$ \\
\hline $\mathrm{K}$ & $4000 \mathrm{Bit}$ \\
\hline $\mathrm{N}$ & 100 \\
\hline
\end{tabular}

The proposed algorithm is compared with LEACH, considering the same parameters of LEACH as shown in Table.1. Figure 2 shows, proposed algorithm in which the first death node occurs later than LEACH and the network life is prolonged by $150 \%$. The sensors are simulated to deploy over a square sized area of $100 \mathrm{~m} \times 100 \mathrm{~m}$ with variable communication range. Simulation is performed using Mat Lab. Figure 2 illustrates the performance comparison of proposed algorithm and LEACH in terms of network lifetime.

Also the performance comparison of proposed Algorithm and LEACH in terms of energy consumption is shown in Figure 3; energy consumption of proposed Algorithm is less when compared to LEACH protocol in all cases. Thus it is energyefficient and has optimum performance when compared to LEACH. Simulation shows our proposed algorithm can balance the energy consumption of the entire network compared to LEACH protocol. Figure 4 shows; the throughput of proposed algorithm is increased by $150 \%$. Though in the beginning period, the throughput is same as LEACH, after more than 1200 rounds, the throughput of proposed algorithm will exceed LEACH greatly.
As shown in Figure 5 number of packets received at the BS per round in the case of total number of nodes being 100, the initial energy being different, and also Figure 5, proposed algorithm has better performance of data transmission to the BS.

Figure 6 shows number of packets received at the $\mathrm{CH}$ per round in the case of total number of nodes being 100 and initial energy being different. From Figure 6, proposed algorithm has better performance of data transmission to the $\mathrm{CH}$ (see Figure 6.).

Figure 7 shows, dead nodes will appear much later in our proposed algorithm compared to LEACH.

Where:

Eelect: Energy consumed in the electronics circuit to transmit or receive the Signal;

Efs: Energy consumed by the amplifier to transmit to a short distance;

Eamp: Energy consumed by the amplifier to transmit to a longer distance;

Eo: Initial energy of each node;

EDA: Data Aggregation Energy;

K: Message Size.

$\mathrm{N}$ : Number of nodes.

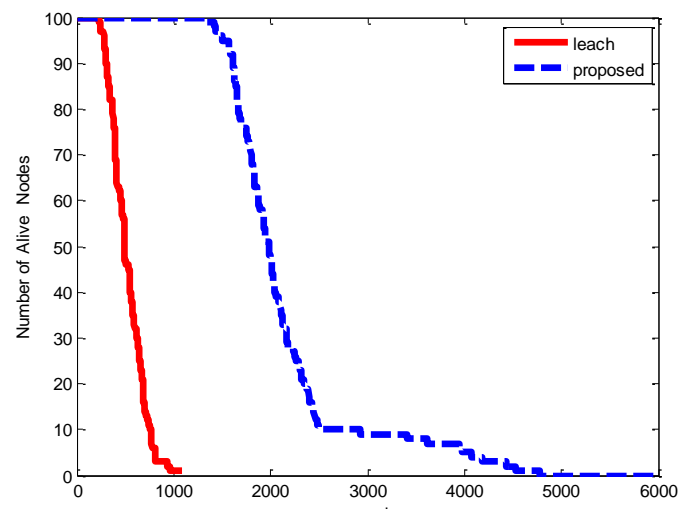

Fig .2 Numbers of alive nodes

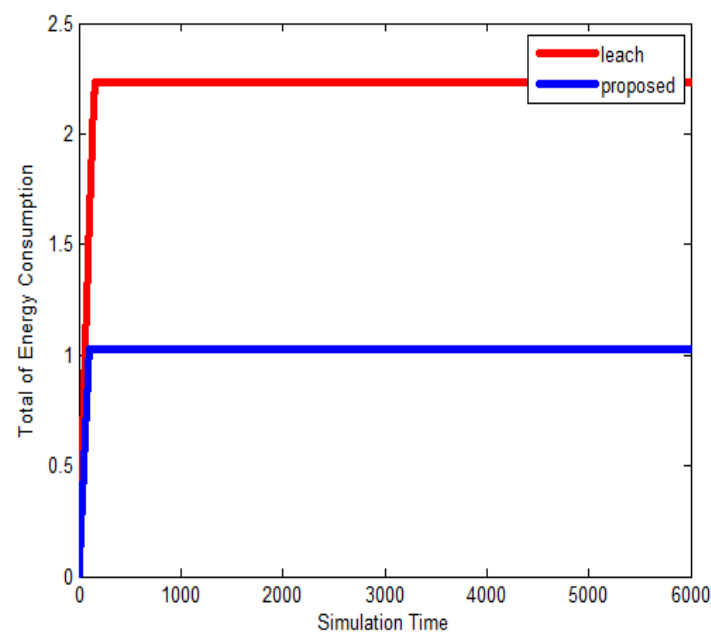

Fig.3 Energy consumption 


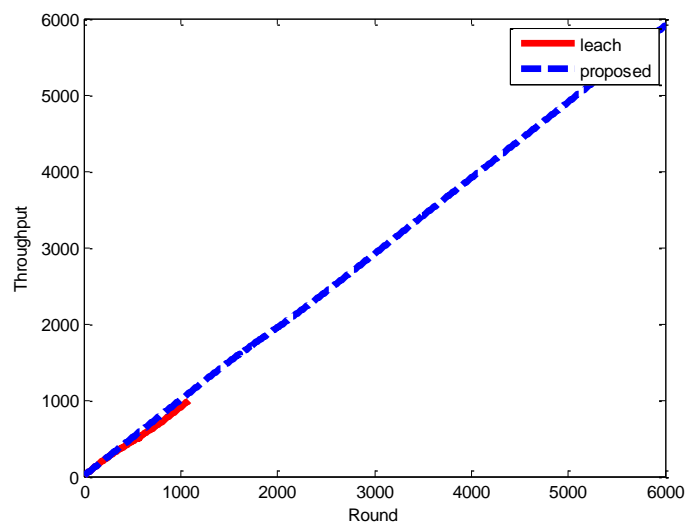

Fig .4 Comparison of number of throughput

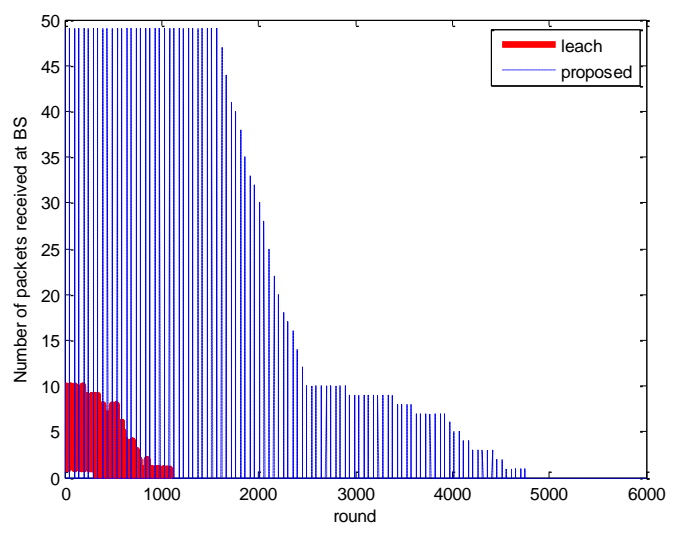

Fig .5 Number of packet received at the BS (different

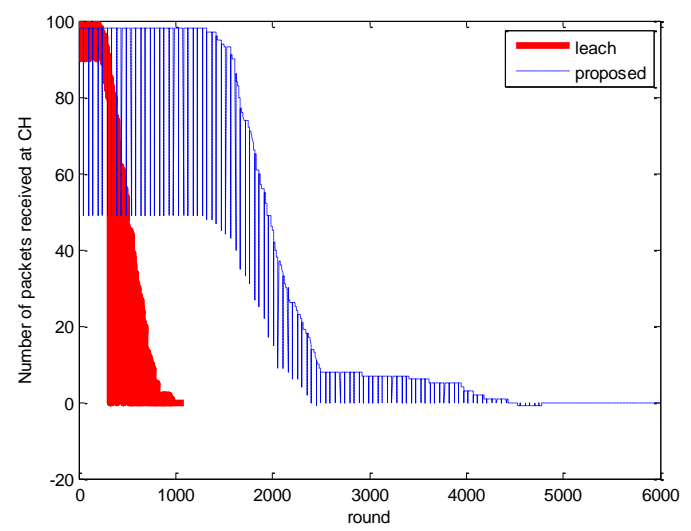

Fig .6 Number of packet received at the $\mathrm{CH}$ (different initial energy and total number of nodes 100)

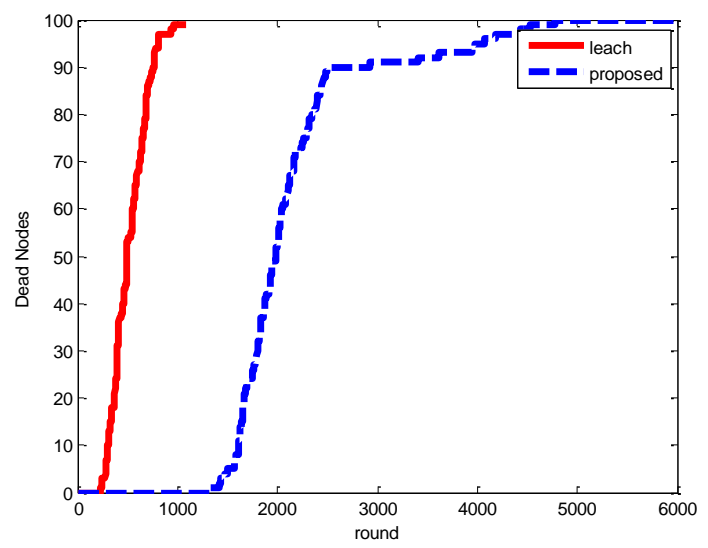

Fig .7 Dead nodes in 100*100 area.

\section{CONCLUSIONS}

In this paper, we have selects three nodes with the highest energy among the nodes deployed, as cluster heads. Each one of the three cluster heads build a set of nodes from the surrounding nodes, where the surrounding nodes with the cluster heads, formed a balanced binary tree, containing high-energy and lowenergy at the same time. Each one of the three cluster heads gathered data from the surrounding nodes and send it to the base station. Proposed algorithm ensures that if nodes have different amounts of energy, then the nodes with more energy should be cluster heads more often than the nodes with less energy. We showed that in many cases proposed algorithm is more energy efficient than LEACH. Our simulation results show that the proposed algorithm provides better energy efficiency and longer network lifetime than the LEACH.

\section{ACKNOWLEDGEMENTS}

The authors wish to acknowledge J.S.S Research Foundation, S.J.C.E Technical institutions campus, Mysore, Karnataka, India for all the facilities provided for this research work.

\section{REFERENCES}

[1] Li Xunbo, Li Na, Chen Liang, Shen Yan, Wang Zhenlin, and Zhu Zhibin"An Improved LEACH for Clustering Protocols In Wireless Sensor Networks". 2010 International Conference on Measuring Technology and Mechatronics Automation.

[2] Edgar H. Callaway Jr. "Wireless Sensor Networks Architectures and Protocols". BeiJing: Electronic Industry Press, 2007, pp.2-12.

[3] W.Heinzelman, A.Chandrakasan, H. Balakrishnan. "Energy-Efficient communication protocol for wireless microsensor network", Proc. of the Hawaii International Conference on System Sciences, IEEE Computer Society, Washington. DC USA, Jan 2000, pp.3005-3014.

[4] S. Lindsey, C. S. Raghavendra, "PEGASIS: PowerEfficient gathering in sensor information systems". In: Proc. of the IEEE Aerospace Conference, Big, Sky, Montana, July 2002, vol.3, pp.1125-1130

[5] A. Manjeshwar and D. P. Agarwal, "TEEN: a routing protocol for enhanced efficiency in wireless sensor networks", In proceedings of the 1st International Workshop on Parallel and Distributed Computing Issues in Wireless Networks and Mobile Computing, IEEE Computer Society, San Francisco, April 2001, pp.20092015. 
[6] W. B. Heinzelman, "Application-Specific Protocol Architectures for Wireless Networks."Ph.D. Dissertation, Department of Electrical Engineering and Computer Science, MIT, Cambridge, MA, June 2000.

[7] Georgios Smaragdakis Ibrahim Matta Azer Bestavros" SEP: A Stable Election Protocol for clustered heterogeneous wireless sensor networks" Technical Report BUCS-TR-2004.

[8] B. Li, Q. Wang, Y. Yang and J. Wang, "Optimal Distribution of Redundant Sensor Nodes for Wireless Sensor Networks," Proc. IEEE Intl.Conf. On Industrial Informatics, 2006, pp. 985 - 989.

[9] A. Chamam and S. Pierre, "Energy-Efficient State Scheduling for Maximizing Sensor Network Lifetime under Coverage Constraint, "Wireless and Mobile Computing, Networking and Communications, 2007, pp. $63-63$.

[10] L. Ren, Z. Guo and R. Ma, "Prolonging Sensor Network Lifetime with Initial Energy Level Assignment," Software Engineering, Artificial Intelligence, Networking, and Parallel/Distributed Computing, 2008, pp.231 - 236.

[11] Guilin, P. R. China"ESCAL: An Energy-Saving Clustering Algorithm Based on LEACH” 978-1-4244-3531-9/082008 IEEE

[12] M. Hasegawa, T. Kawamura, H. N. Tran, G. Miyamoto, Y. Murata, H. Harada and S. Kato, "Decentralized optimization of wireless sensor network lifetime based on neural network dynamics," Proc. IEEE Intl. Conf. on Personal, Indoor and Mobile Radio Communications (PIMRC),2008, p. 1.

[13] CHEN Jian-ming,LU Jian-jun , WANG Qing-hai "Research and Improvement of Adaptive Topology Algorithm LEACH for Wireless Sensor Network" 978-14244-2108-4/08/ 2008 IEEE

[14] Hu Junping, Jin Yuhui, Dou Liang“"A Time-based ClusterHead Selection Algorithm for LEACH” 978-1-4244-27031/08[35]Hanady M. Abdulsalam, Layla K. Kamel"WLEACH: Weighted Low Energy Adaptive Clustering Hierarchy Aggregation Algorithm for Data Streams in Wireless Sensor Networks" 2010 IEEE International Conference on Data Mining Workshops./2008 IEEE

[15] N. H. Mak and W. K. G. Seah, "How Long s the Lifetime of a Wireless Sensor Network?" Proc. IEEE Intl. Conf. on Advanced Information Networking and Applications (AINA), 2009, p. 763

[16] Chong Wang, Jiakang Liu, Jingming Kuang, Abdul Sattar Malik, Huihui Xiang, "An Improved LEACH Protocol for Application-Specific Wireless Sensor Networks" 978-14244-3693-4/09/2009 IEEE

[17] Tang qiang, Wang bingwen, Dai zhicheng, "MS-Leach: A Routing Protocol Combining Multi-hop Transmissions and Single-hop Transmissions" 2009 Pacific-Asia Conference on Circuits,Communications and System

[18] S. Xiong, J. Li and L. Yu, "Maximize the Lifetime of a Data-gathering Wireless Sensor Network," Proc. IEEE Intl. Conf. on Sensor, Mesh and Ad Hoc Communications and Networks (SECON), 2009, p. 1.

[19] Li Xunbo, Li Na, Chen Liang, Shen Yan, Wang Zhenlin, Zhu Zhibin“An Improved LEACH For Clustering
Protocols In Wireless Sensor Networks" 2010 International Conference on Measuring Technology and Mechatronics Automation

[20] WU Xiaoping, LIN Hong, LI Gang”An Improved Routing Algorithm Based On LEACH Protocol” 2010 Ninth International Symposium on Distributed Computing and Applications to Business, Engineering and Science. 978-07695-4110-5/10 / 2010 IEEE

[21] Yaqiong Wang, Qi Wang, Ziyu Jin "Improved Cluster Heads Selection Method in Wireless Sensor Networks" 2010 IEEE/ACM International Conference on Green Computing and Communications \& 2010 IEEE/ACM International Conference

[22] Lu Tao, Zhu Qing-Xin1, Zhang Luqiao"An Improvement for LEACH Algorithm in Wireless Sensor Network" 9781-4244-5046-6/10/ 2010 IEEE

[23] Gao JingMin, Zeng Zhiliang, Gaoyang"Research and improvement of routing protocol for wireless sensor network" 2010 International Conference on Computer, Mechatronics, Control and Electronic Engineering (CMCE).

[24] Mu Tong, Minghao Tang,"LEACH-B:An Improved LEACH Protocol for Wireless Sensor Network" 978-14244-3709-2/10/2010 IEEE

[25] Haosong Gou and Younghwan Yoo,"An Energy Balancing LEACH Algorithm for Wireless Sensor Networks" 2010 Seventh International Conference on Information Technology

[26] Fatemeh Ayughi", Karim Faez**, Zahra Eskandarf"A non location aware version of modified LEACH algorithm based on Residual Energy and Number of Neighbors" Feb. 7-10, 2010 ICACT 2010

[27] LIHan, P.R.China"LEACH-HPR: An Energy Efficient Routing Algorithm for Heterogeneous WSN" 978-1-42446585-9/10/2010 IEEE

[28] Muhamnmad Omer Farooq, Abdul Basit Dogar, Ghalib Asadullah Shah "MR-LEACH: Multi-hop Routing with Low Energy Adaptive Clustering Hierarchy" 2010 Fourth International Conference on Sensor Technologies and Applications 978-0-7695-4096-2/ 2010 IEEE

[29] D. Kumar, T.C. Aseri, R.B. Patel,” EECDA: Energy Efficient Clustering and Data Aggregation Protocol for Heterogeneous Wireless Sensor Networks", Int. J. of Computers, Communications \& Control, ISSN 1841-9836, E-ISSN 1841-9844 Vol. VI (2011), No. 1 (March), pp. 113-124

[30] Vivek Katiyar"Improvement in LEACH Protocol for Large-scale Wireless Sensor Networks"Department of Computer Science \& Engineering National Institute of Technology Hamirpur, HP, INDIA, 978-1-4244-79269/11/2011 IEEE

[31] Naveen Kumar, Mrs. Jasbir Kaur'Improved LEACH Protocol for Wireless Sensor Networks" 978-1-4244-62520/2011 IEEE

[32] Bilal Abu Bakr, Leszek Lilien,"A Quantitative Comparison of Energy Consumption and WSN Lifetime for LEACH and LEACH-SM" 201131 st International Conference on Distributed Computing Systems Workshops. 15450678/11/2011 IEEE 
[33] A. Rahmanian, H. Omranpour, M. Akbari, K. Raahemifar "A Novel Genetic Algorithm In LEACH-C Routing Protocol For Sensor Networks" IEEE CCECE 2011 001100

[34] T. Anker, D. Bickson, D. Dolev, and B. Hod, Efficient Clustering for Improving Network Performance in Wireless Sensor Networks, in Proceedings of the Springer -Verilag Heridelberg (LNCS 4913),221-236, 2008.

[35] M. J. Handy, M. haase, D. Timmermann. Low energy adaptive clustering hierarchy with deterministic clusterhead selection[C]. Proc. of the 4th IEEE Conf. on Mobile and Wireless Communication Network, Stockholm, 2002: $368 \sim 372$.

[36] P. Tillapart, T. Thumthawatwon, P. Pakedeepinit, T. Yeophantong, S. Charoenvikrom, J. Daengdej. Method for cluster heads selection in wireless sensor networks[C]. Proc. Of the 2004 IEEE Aerospace Conference, Chiang Mai, 2004:3615 3623.

[37] W. Heinzelman, A. Chandrakasan and H.Balakrishnan,"Energy-Efficient Communication Protocol for Wireless Micro-sensor Networks," Proceedings of the 33rd Hawaii International Conference on System Sciences (HICSS '00), January. 2000.

[38] Udit Sajjanhar and Pabitra Mitra. Distributive energy efficient adaptive clustering protocol for wireless sensor networks. In: Proceedings of IEEE International Conference on Advanced Information Networking and Application, 2006.

\section{AUTHOR PROFILE}

Mr.Abdo Saif Mohammed Completed his B.Sc. degree in Computer Science \& Information System from University of Technology-IRAQ in the year 2001, M.Sc. in Computer Communication from Bharathiar University-India in the year 2009. And $\mathrm{He}$ is presently working in the Department of Computer Science, Thamar University Yemen, Dhamar, Yemen. He is doing his $\mathrm{Ph} . \mathrm{D}$ in the area of Wireless sensor networks under the guidance of Dr. M.N Shanmukhaswamy.

Dr.M.N.Shanmukha Swamy completed his B.E. degree in Electronics and Communication from Mysore University in the year 1978, M.Tech in Industrial Electronics from the same university in the year 1987 and obtained his $\mathrm{PhD}$ in the field of Composite materials from Indian Institute of Science, Bangalore in 1997. He is presently working as Professor in the Department of Electronics and communication, Sri Jayachamarajendra college of Engineering, Mysore, Karnataka, India. He is guiding several research scholars and has published many books \& papers both in National \& International conferences \& journals. His research area includes Wireless Sensor Networks, Biometrics, VLSI and composite materials for application in electronics. 